\title{
Medical Robots: Current Systems and Research Directions
}

\author{
Ryan A. Beasley \\ Department of Engineering Technology and Industrial Distribution, Texas A\&M University,
} 3367 TAMU, College Station, TX 77843, USA

Correspondence should be addressed to Ryan A. Beasley, beasley@entc.tamu.edu

Received 10 March 2012; Revised 19 June 2012; Accepted 6 July 2012

Academic Editor: Farrokh Janabi-Sharifi

Copyright (C) 2012 Ryan A. Beasley. This is an open access article distributed under the Creative Commons Attribution License, which permits unrestricted use, distribution, and reproduction in any medium, provided the original work is properly cited.

First used medically in 1985, robots now make an impact in laparoscopy, neurosurgery, orthopedic surgery, emergency response, and various other medical disciplines. This paper provides a review of medical robot history and surveys the capabilities of current medical robot systems, primarily focusing on commercially available systems while covering a few prominent research projects. By examining robotic systems across time and disciplines, trends are discernible that imply future capabilities of medical robots, for example, increased usage of intraoperative images, improved robot arm design, and haptic feedback to guide the surgeon.

\section{Introduction}

Medical robotics is causing a paradigm shift in therapy. The most widespread surgical robot, Intuitive Surgical's da Vinci system, has been discussed in over 4,000 peer-reviewed publications, was cleared by the United States' Food and Drug Administration (FDA) for multiple categories of operations, and was used in $80 \%$ of radical prostatectomies performed in the U.S. for 2008, just nine years after the system went on the market [1-3]. The rapid growth in medical robotics is driven by a combination of technological improvements (motors, materials, and control theory), advances in medical imaging (higher resolutions, magnetic resonance imaging, and 3D ultrasound), and an increase in surgeon/patient acceptance of both laparoscopic procedures and robotic assistance. New uses for medical robots are created regularly, as in the initial stages of any technology-driven revolution.

In 1979, the Robot Institute of America, an industrial trade group, defined a robot as "a reprogrammable, multifunctional manipulator designed to move materials, parts, tools, or other specialized devices through various programmed motions for the performance of a variety of tasks." Such a definition leaves out tools with a single task (e.g., stapler), anything that cannot move (e.g., image analysis algorithms), and nonprogrammable mechanisms (e.g., purely manual laparoscopic tools). As a result, robots are generally indicated for tasks requiring programmable motions, particularly where those motions should be quick, strong, precise, accurate, untiring, and/or via complex articulations. The downsides generally include high expense, space needs, and extensive user training requirements. The greatest impact of medical robots has been in surgeries, both radiosurgery and tissue manipulation in the operating room, which are improved by precise and accurate motions of the necessary tools. Through robot assistance, surgical outcomes can be improved, patient trauma can be reduced, and hospital stays can be shortened, though the effects of robot assistance on long-term results are still under investigation.

Medical robots have been reviewed in various papers since the 1990s [4-7]. Many such reviews are domainspecific, for example, focusing on surgical robots, urological robots, spine robots, and so forth [8-13]. For an overview of the basic science behind medical robots (e.g., kinematics, degrees of freedom, ergonomics, and telesurgery) along with a discussion of urologic robotic systems, see Challacombe and Stoianovici [14]. Similarly focused on surgery, Kenngott et al. provide a recent Medline metareview on the outcomes of laparoscopic robot-assisted surgeries (urologic, gynecologic, and abdominal) [15], while Gomes covers market drivers and roadblocks [16], and Okamura et al. explore big picture issues like societal drivers, quantitative diagnosis, and system adaptation/learning [17]. The most recent coverage of medical robots across various domains was by Najarian et al. and the articles collected by Rosen et al. [18, 19]. 
This paper provides an overview of the impact of robots in multiple medical domains. This work builds on top of the aforementioned papers by providing an updated review of various robotic systems, covering system improvements (technical and regulatory) and changes in manufacturers due to corporate buyouts. Furthermore, to the author's knowledge this work covers more breadth in the medical domains benefiting from robot assistance than any other single paper, and thus provides a big picture view of how robots are improving the medical field. Though primarily focused on commercially available medical robotic systems and the history that describes their evolution, this paper also covers multiple next-generation systems and discusses their potential impacts on the future of the medical field.

\section{Neurological}

Brain surgery involves accessing a buried target surrounded by delicate tissue, a task that benefits from the ability for robots to make precise and accurate motions based on medical images [18, 20,21]. Thus, the first published account investigating the use of a robot in human surgery was in 1985 for brain biopsy using a computed tomography (CT) image and a stereotactic frame [22]. In that work, an industrial robot defined the trajectory for a biopsy by keeping the probe oriented toward the biopsy target even as the surgeon manipulated the approach. This orientation was determined by registering a preoperative CT with the robot via fiducials on a stereotactic frame attached to the patient's skull. That project was discontinued after the robot company was bought out, due to safety concerns of the new owning company, which specified that the robot arm ( $54 \mathrm{~kg}$ and capable of making $0.5 \mathrm{~m} / \mathrm{s}$ movements) was only designed to operate when separated by a barrier from people. Then in 1991, the Minerva robot (University of Lausanne, Switzerland) was designed to direct tools into the brain under real-time CT guidance. Real-time image guidance allows tracking of targets even as the brain tissue swells, sags, or shifts due to the operation. Minerva was discontinued in 1993 due to the limitation of single-dimensional incursions and its need for real-time CT [23].

The currently available neurosurgery robots exhibit a purpose similar to historical systems, namely, image-guided positioning/orientation of cannulae or other tools (Figure 1). The NeuroMate (by Renishaw, previously by Integrated Surgical Systems, previously by Innovative Medical Machines International) has a Conformité Européenne (CE) mark and is currently used in the process for FDA clearance (the previous generation was granted FDA clearance in 1997) [24]. In addition to biopsy, the system is marketed for deep brain stimulation, stereotactic electroencephalography, transcranial magnetic stimulation, radiosurgery, and neuroendoscopy. Li et al. report in-use accuracy as submillimeter for a frame-based configuration, the same level of application accuracy as bone-screw markers with infrared tracking, and an accuracy of $1.95 \mathrm{~mm}$ for the frameless configuration [25].

Another robotic system, Pathfinder (Prosurgics, formerly Armstrong Healthcare Ltd.), has been cleared by the FDA for neurosurgery (2004) [26]. Using the system, the surgeon specifies a target and trajectory on a pre-operative medical image, and the robot guides the instrument into position with submillimeter accuracy [27]. Reported uses of the system include guiding needles for biopsy and guiding drills to make burr holes [28].

Renaissance (Mazor Robotics, the first generation system was named SpineAssist) has FDA clearance (2011) and CE mark for spinal surgery, and a CE mark for brain operations (2011) [29]. The device consists of a robot the size of a soda can that mounts directly onto the spine and provides tool guidance based on planning software for various procedures including deformity corrections, biopsies, minimally invasive surgeries, and electrode placement procedures. Renaissance includes an add-on for existing fluoroscopy C-arms that provides 3D images for intraoperative verification of implant placement. Studies show increased implant accuracy and provide evidence that the Renaissance/SpineAssist may allow significantly more implants to be placed percutaneously [30].

\section{Orthopedics}

The expected benefit of robot assistance in orthopedics is accurate and precise bone resection $[31,32]$. Through good bone resection, robotic systems (Figure 2) can improve alignment of implant with bone and increase the contact area between implant and bone, both of which may improve functional outcomes and implant longevity [5]. Orthopedic robots have so far targeted the hip and knee for replacements or resurfacing (the exception being the Renaissance system in Section 2 and its use on the spine). Initial systems required the bones to be fixed in place, and all systems use bone screws or pins to localize the surgical site.

The initial robot assistance for orthopedics came via Robodoc (Curexo Technology Corp, originally by Integrated Surgical Systems), first used in 1992 for total hip replacement $[5,33]$. Robodoc has received a CE mark (1996), and FDA clearance for total hip replacement (1998) and total knee replacement (2009) [34]. The robot is used in conjunction with OrthoDoc, a surgical planner, with which the surgeon plans bone milling is based on preoperative CT. During the procedure, the patient's leg is clamped to the robot's pedestal, and a second clamp locates the femoral head to automatically halt the robot if the leg moves. The Robodoc then performs the milling automatically based on the surgical plan. Many initial attempts in surgical robotics involved such autonomous motions, which generated concerns about patient and doctor safety. To address those concerns, Robodoc has force sensing on all axes, as well as a six-axis force sensor at the wrist [35]. The force sensing is used for safety monitoring, to allow the surgeon to manually direct the robot arm and to vary the velocity of tool motion as a function of the forces experienced during the milling operation.

Though no longer for sale, CASPAR (Computer Assisted Surgical Planning and Robotics) was another robotic system for knee and hip surgery, introduced in 1997 by OrtoMaquet, 


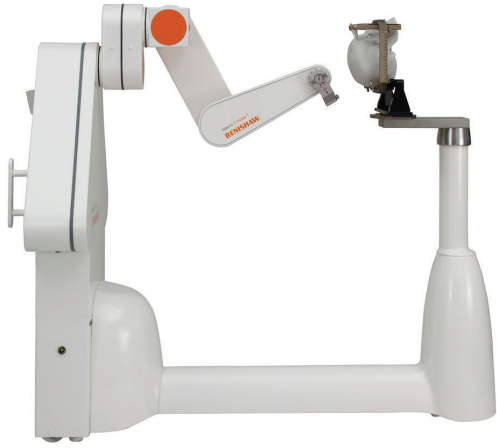

(a) NeuroMate by Renishaw

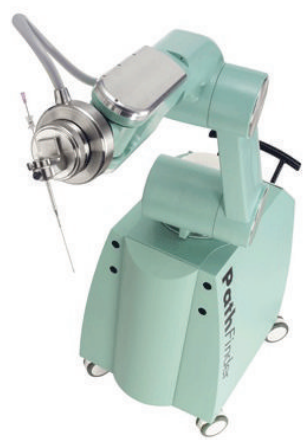

(b) Pathfinder by Prosurgics

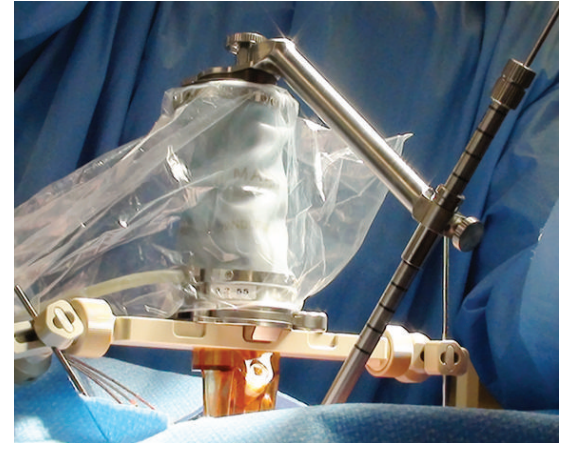

(c) Renaissance by Mazor Robotics

Figure 1: Neurosurgery robots for image-guided tool positioning/orientation. The NeuroMate image is @2012 Renishaw. The Pathfinder image is ( 2012 Prosurgics. The Renaissance image is (2011 Mazor Robotics Ltd. All rights reserved with nonexclusive permission.

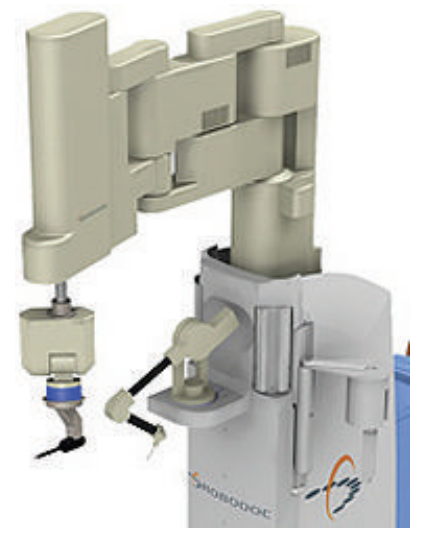

(a) Robodoc by Curexo Technology Corp.

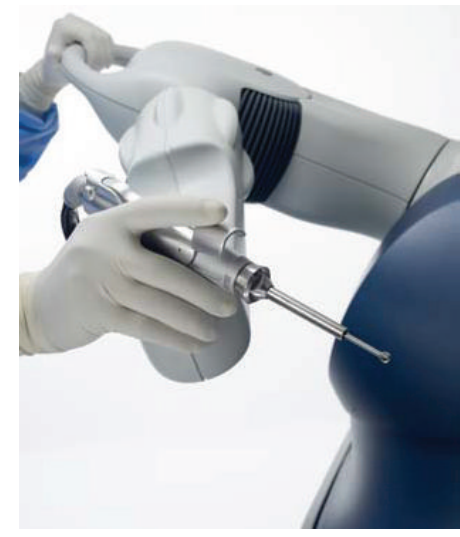

(b) RIO by MAKO Surgical Corp.

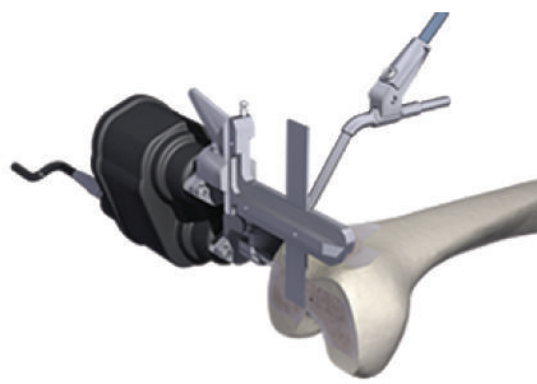

(c) iBlock by Praxim Inc.

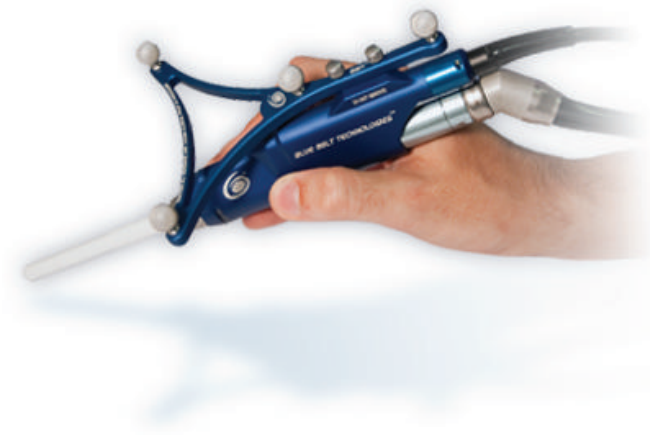

(d) Navio PFS by Blue Belt Tech.

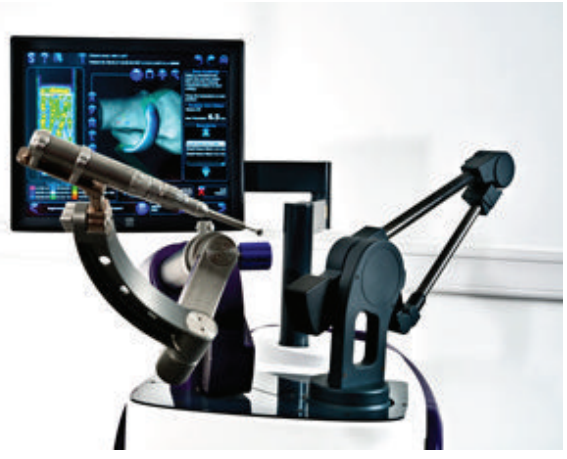

(e) Stanmore Sculptor by Stanmore Implants

FIgURe 2: Orthopedic robots for accurate bone resection. The Robodoc image is (C2012 Curexo Technology Corp. The RIO image is $\odot 2012$ MAKO Surgical Corp. The iBlock image is (2012 Praxim Inc. The Navio PFS image is (2012 Blue Belt Tech. The Stanmore Sculptor image is (2012 Stanmore Implants.

acquired by Getinge in 2000, acquired and discontinued by Universal Robot Systems (URS) in 2001. The robot was a direct competitor to Robodoc. It automatically performed bone drilling from a preoperative plan based on CT data.

In 2008, the RIO robotic arm (MAKO Surgical Corp, previous generation called the Tactile Guidance System) was released and received FDA clearance. The RIO is used for implantation of medial and lateral unicondylar knee components, as well as for patellofemoral arthroplasty [36, 37]. As part of the trend away from autonomous robot motions, both the RIO and the surgeon simultaneously hold the surgical tool, with which the surgeon moves about the 
surgical site. The arm is designed to be low friction and low inertia, so that the surgeon can easily move the tool, backdriving the arm's joint motors in the process [19]. The arm's purpose is to act as a haptic device during the milling procedure, resisting motions outside of the planned cutting envelope by pushing back on the surgeon's hand. Unlike other orthopedic systems, the RIO does not require the bone to be fixed in place, instead relying on a camera system to track bone pins and tools intraoperatively and instantaneously registering the planned cutting envelope to the patient in the operating room. With this configuration, the system has promise for use as a surgical training tool.

Further reducing robotic influence on the cutting tool, the iBlock (Praxim Inc., an Orthopaedic Synergy Inc. company, previous generation the Praxiteles, FDA clearance 2010 ) is an automated cutting guide for total knee replacement [38]. The iBlock is mounted directly to the bone, preventing any relative motion between the robot and the bone and aligns a cutting guide that the surgeon uses to manually perform planar cuts based on a preoperative plan. Koulalis et al. report reduced surgical time and increased cut accuracy compared with freehand navigation of cutting blocks [39].

The Navio PFS (Blue Belt Technologies, CE mark 2012) does not require a CT scan for unicondylar knee replacement, instead it uses intraoperative planning $[40,41]$. The drill tool is tracked during the procedure, and the drill bit is retracted when it would leave the planned cutting volume. Limited information is available on the system due to its recent development.

The Stanmore Sculptor (Stanmore Implants, previous generation the Acrobot Sculptor by Acrobot Company Ltd.) is a synergistic system similar to the RIO, with active constraints to keep the surgeon in the planned workspace [42]. The company's "Savile Row" system tailors a personalized unicondylar knee implant to the patient, incorporates the 3D model of that implant into the surgical planning interface, and uses active constraints with the Stanmore Sculptor to ensure proper preparation of the bone surface. The system does not currently have FDA clearance, but has been in use in Europe since 2004.

\section{General Laparoscopy}

Prior to the 1980s, surgical procedures were performed through sizable incisions through which the surgeon could directly access the surgical site. In the late 1980s, camera technology had improved sufficiently for laparoscopy (a.k.a. minimally invasive surgery), in which one or more small incisions are used to access the surgical site with tools and camera [43]. Laparoscopy significantly reduces patient trauma in comparison with traditional "open" procedures, thereby reducing morbidity and length of hospital stay, but at the cost of increased complexity of the surgical task. Compared with open surgery, in laparoscopy the surgeon's feedback from the surgical site is impaired (reduced visibility and cannot manually palpate the tissue) and tool control is reduced ("mirror-image" motions due to fulcrum effect and loss of degrees of freedom in tool orientation) $[16,44,45]$.

Robot assistance for soft-tissue surgery was first done in 1988 using an industrial robot to actively remove soft tissue during transurethral resection of the prostate [5]. As with neurosurgery, the researchers deemed use of an industrial robot in the operating room to be unsafe. The experience provided the impetus for a research system, Probot, with the same purpose [46].

4.1. Zeus. Commercial robotic systems for laparoscopy started with Computer Motion's Aesop (discontinued, FDA clearance 1993) for holding endoscopes [47]. Aesop was clamped to the surgical table or to a cart, and either moved the endoscope under voice control or allowed the endoscope to be manually positioned. In 1995, Computer Motion combined two tool-holding robot arms with Aesop to create the Zeus system (discontinued, FDA clearance 2001) [48]. The Zeus's tool arms were teleoperated, following motions the surgeon made with instrument controls (a.k.a. "master" arms or joysticks) at the surgeon console. Technically, the Zeus is not a robot because it does not follow programmable motions, but rather is a remote computer-assisted telemanipulator with interactive robotic arms. To improve precision in tool motion, the Zeus filters out hand tremor, and can scale large hand motions by the surgeon down to short and precise motions by the tool. As described by Marescaux et al., the Zeus was used in the Lindbergh Operation, the first surgery was (cholecystectomy) performed with the surgeon and patient being separated by a distance of several thousand kilometers [49].

4.2. da Vinci. Meanwhile, Intuitive Surgical Inc. was developing the da Vinci (initial FDA clearance 1995, Figure 3(a)). Like the Zeus, the da Vinci is a teleoperated system, wherein the surgeon manipulates instrument controls at a console and the robot arms follow those motions with motion scaling and tremor reduction. Also like the Zeus, the da Vinci was initially offered with three arms to hold two tools and an endoscope, which are mounted to a single bedside cart.

The da Vinci system provides several technical enhancements over the Zeus. The grasper tools have two degrees of freedom inside the patient, the EndoWrist (Figure 3(b)), an enhanced articulation that increases the ease of suturing and other complex manipulations. The console puts increased emphasis on surgeon ergonomics and incorporates a separate video screen for each eye to display $3 \mathrm{D}$ video from the $3 \mathrm{D}$ endoscope. The motions of the surgeon's hands are mapped to motions of the operational ends of the tools, providing a more intuitive control than the "mirror-image" laparoscopic mapping. In 2003, Intuitive Surgical began selling a fourth arm for the da Vinci, and Intuitive Surgical and Computer Motion were merged (discontinuing the Zeus).

The da Vinci system is the only surgical robot with over a thousand systems installed worldwide and has been sold in four models so far: Standard (1999), S (2006), Si (2009), and Si-e (2010) [50, 51]. The S model increased the image resolution, redesigned the patient-side manipulators 


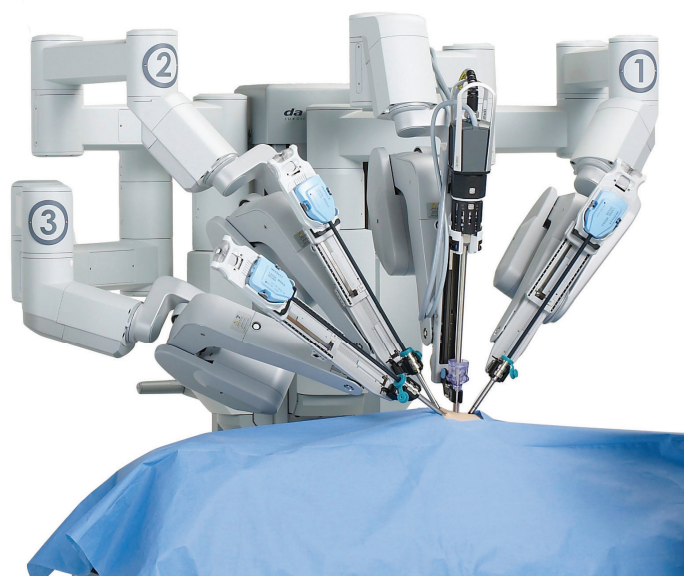

(a) Da Vinci Si patient-side cart

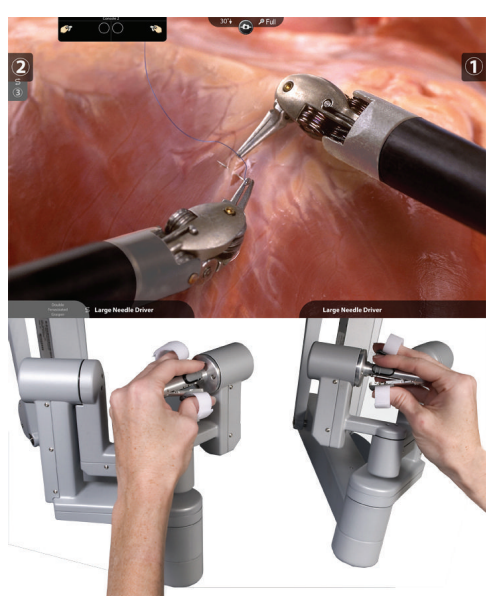

(b) Da Vinci EndoWrist and controllers

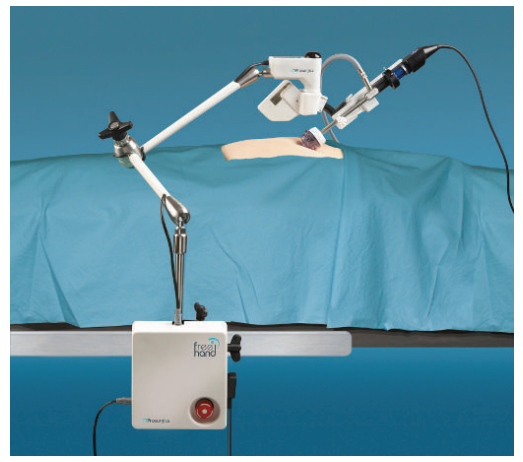

(c) FreeHand by Freehand 2010 Ltd.

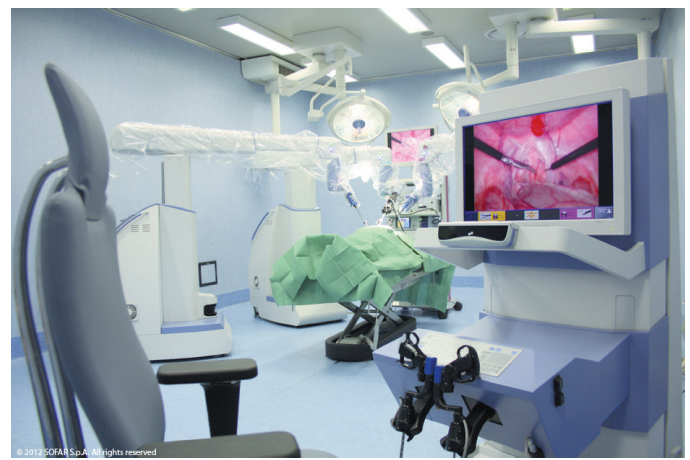

(d) Telelap ALF-X by SOFAR S.p.A

FIgURE 3: Laparoscopy robots. The da Vinci Si, by Intuitive Surgical Inc. (a) Cart and (b) image mosaic showing the tool tips with EndoWrist articulation, and the instrument controls. (c) FreeHand, a next-generation endoscope holder. (d) A computer model of the Telelap ALF-X, by SOFAR S.p.A. The da Vinci images are ( 2012 Intuitive Surgical, Inc. The FreeHand image is @2012 Freehand 2010 Ltd. The Telelap ALF-X image is (2012 SOFAR S.p.A. All rights reserved.

to enable multiquadrant access, and shortened setup time. The Si model further improved the visual resolution, refined the instrument controllers, and increased the ergonomics and ease for the surgeon to provide input to the system. The Si-e model is a 3 -arm system that is fully upgradeable to the Si model. Continuing the da Vinci focus on improved visualization, the Firefly Fluorescence Imaging add-on product combines fluorescent dye and a special endoscope to identify vasculature beneath the tissue surface.

The da Vinci was initially cleared for general laparoscopy, became commonly used for radical prostatectomy, and is now cleared by the FDA for various procedures [52, 53]. Even so, as with most or even all robotic systems, long-term benefits continue to be uncertain $[15,54]$. The enhanced endoscopic visualization and increased tool articulation are commonly considered improvements, but detractors point out the system's expense (between $\$ 1 \mathrm{M}$ and $\$ 2.3 \mathrm{M}$ ), the reduced patient access due to the amount of space the arms take over/around the patient, and the significant amount of training necessary for the best outcomes $[55,56]$. To address this last point, the Si model also allows dual console use for training and collaboration, in which both consoles get the same images and can cooperatively control the instruments [57]. Additionally, the da Vinci Skills Simulator is an add-on case that can be used with an Si or Si-e console to practice operations in a virtual environment [58].

In an attempt to further reduce patient trauma, surgeons are exploring Single-Port Access (SPA), LaparoEndoscopic Single-Site surgery (LESS), and Natural Orifice Transluminal Endoscopic Surgery (NOTES) [59, 60]. To meet this need, Intuitive Surgical has recently developed the Single-Site platform for the da Vinci Si model. The Single-Site platform passes two semirigid tools and the endoscope through a single multichannel port, reducing the number of incisions but preventing EndoWrist articulation [61].

4.3. FreeHand. The FreeHand robot (Freehand 2010 Ltd., previously Freehand Surgical, previously Prosurgics, the previous generation was called EndoAssist, FDA clearance and CE mark 2009) is a next-generation endoscope holder. The arm (Figure 3(c)) is more compact, easier to setup, and cheaper than its predecessor. Furthermore, endoscope motion is controlled by gentle head motions by the surgeon, which are tracked with an optical system. 


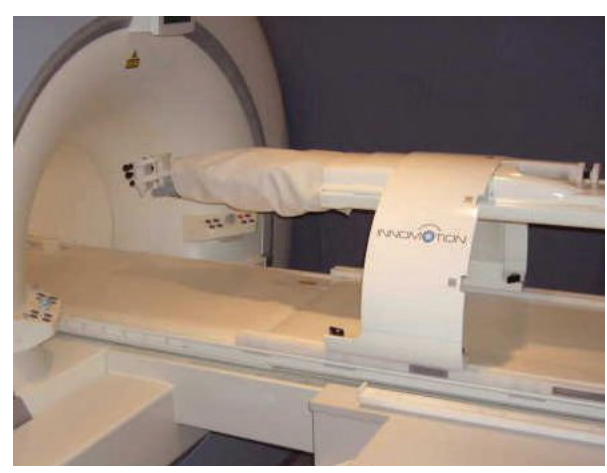

(a) InnoMotion by Synthes Inc.

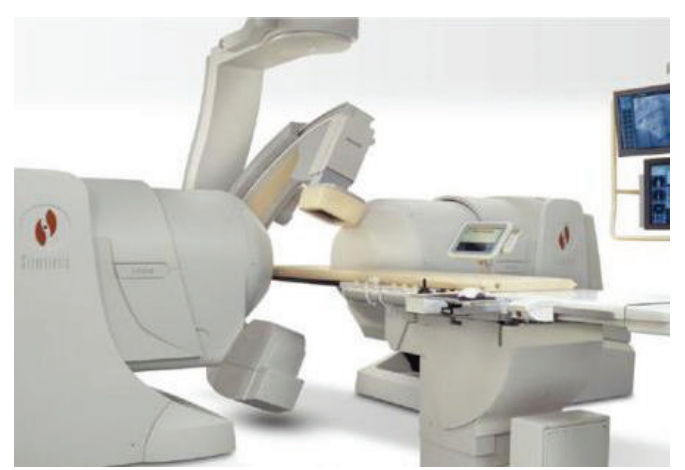

(b) Niobe by Stereotaxis

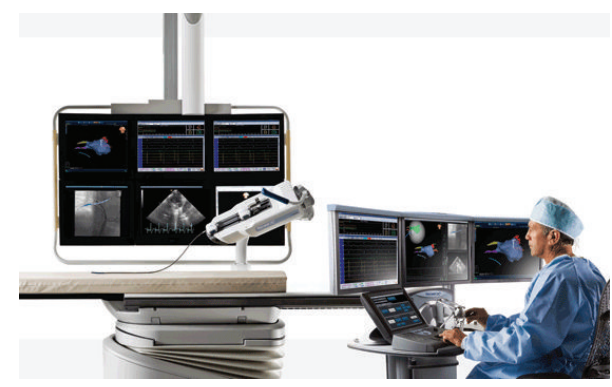

(c) Sensei $\mathrm{X}$ by Hansen Medical

FIgURE 4: Real-time image guided percutaneous (a) and catheter robots ((b) and (c)). The InnoMotion image is @2012 Synthes Inc. The Niobe image is (2012 Stereotaxis. The Sensei X image is (๑2012 Hansen Medical.

4.4. Telelap ALF-X. SOFAR S.p.A has developed Telelap ALFX (CE mark 2011, Figure 3(d)), a four-armed surgical robotic system, to compete with the da Vinci [62]. The system uses eyetracking to control the endoscopic view and to enable activation of the various instruments. Compared to the da Vinci, the system moves the base of the manipulators away from the bed (about $80 \mathrm{~cm}$ ) and has a realistic tactile-sensing capability due to a patented approach to measure tip/tissue forces from outside the patient, with a sensitivity of 35 grams. The system has been used in animal trials demonstrating a significant reduction in the time for cholecystectomy compared with a "conventional telesurgical system" [62].

\section{Percutaneous}

Noncatheter percutaneous procedures employ needles, cannulae, and probes for biopsy, drainage, drug delivery, and tumor destruction. During the procedure, accurate targeting can be reduced by soft tissue displacements that occur due to patient breathing, changes in posture, or tissue forces exerted during the insertion. Two options to guide a needle to its target are tissue modeling for needle steering and threedimensional intraoperative imaging [63]. Unfortunately, tissue modeling is excessively complex [64]. So following the latter approach, InnoMotion (Synthes Inc., previously by Innomedic GmbH, CE mark 2005) is a robot arm designed to operate within a CT or magnetic resonance imaging (MRI) machine [65-67]. For MRI-compatibility, the arm (Figure $4(\mathrm{a})$ ) is pneumatically actuated and joint sensing is via MRI-compatible encoders.

\section{Steerable Catheters}

Vascular catheterization is used to diagnose and treat various cardiac and vasculature diseases, including direct pressure measurements, biopsy, ablation for atrial fibrillation, and angioplasty for obstructed blood vessels [68-70]. The catheter is inserted into a blood vessel and the portion external to the patient is manipulated to move the catheter tip to the surgical site, while fluoroscopy provides image guidance. Due to the supporting tissue, catheters only require three degrees of freedom, typically: tip flexion, tip rotation, and insertion depth. Possible benefits of robotsteered catheters are shorter procedures, reduced forces exerted on the vasculature by the catheter tip, increased accuracy in catheter positioning, and teleoperation (reducing exposure of the physician to radiation) [71].

The Sensei X (Hansen Medical, FDA clearance and CE mark 2007, previous generation the Sensei, Figure 4(c)) uses two steerable sheaths, one inside the other, to create a tight bend radius [72-74]. The sheaths are steered via a remotely operated system of pulleys. IntelliSense force sensing allows constant estimation of the contact forces by gently pulsing the catheter a short distance in and out of the steerable inner sheath and measuring forces at the proximal end of the catheter. These forces are communicated visually as well as through a vibratory feedback to the surgeon's hand on the "3D joystick". Corindus's CorPath 200 is a direct competitor with the Sensei X, but is not yet commercially available.

The Niobe (Stereotaxis, CE mark 2008, FDA clearance 2009) is a remote magnetic navigation system, in which 


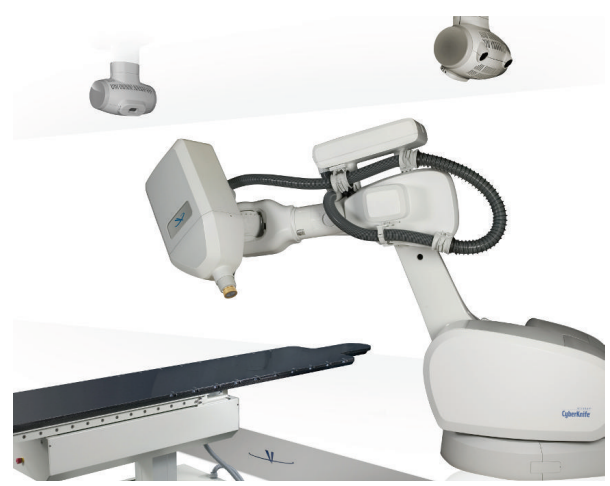

(a) CyberKnife by Accuray Inc.

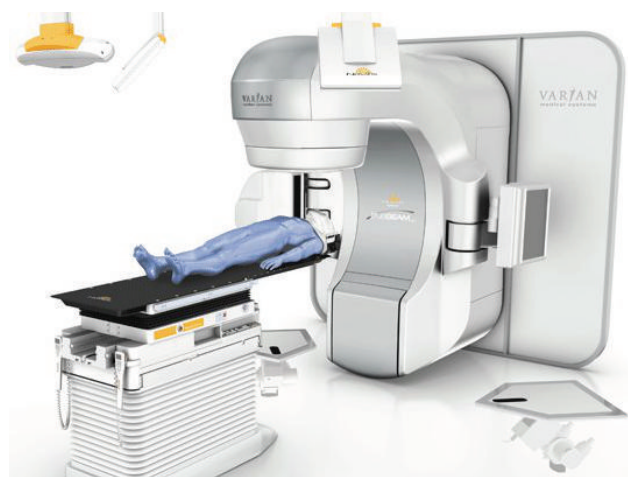

(b) Novalis with TrueBeam STx by BrainLab Inc. and Varian Medical Systems

FIGURE 5: Radiosurgery robots use X-ray images taken during the treatment to control robotic patient tables, ensuring accurate targeting of the radiosurgery beams. The Cyberknife image and any Accuray trademarks or logos are used with permission from Accuray Incorporated. The image of the Novalis with TrueBeam STx is (C2012 BrianLab Inc.

a magnetic field is used to guide the catheter tip [75]. The magnetic field is generated by two permanent magnets contained in housings on either side of a fluoroscopy table (Figure 4(b)). The surgeon manipulates a joystick to specify the desired orientation of the catheter tip, causing the orientations of the magnets to vary under computer-control, and thereby controlling the magnetic field. A second joystick controls advancement/retraction of the catheter. Chun et al. report significant improvements in surgical outcomes due to advances in the design of magnetically guided catheters [76].

\section{Radiosurgery}

Radiosurgery is a treatment (not a surgery), in which focused beams of ionizing radiation are directed at the patient, primarily to treat tumors $[77,78]$. By directing the beam through the tumor at various orientations, high-dose radiation is delivered to the tumor while the surrounding tissue receives significantly less radiation. Prior to realtime tissue tracking, radiosurgery was practically limited to treating the brain using stereotactic frames mounted to the skull with bone screws. Now that real-time tissue tracking is feasible, systems are commercially available.

The CyberKnife (Accuray Inc., FDA cleared 1999, Figure $5(\mathrm{a})$ ) is a frameless radiosurgery system consisting of a robotic arm holding a linear accelerator, a six degree of freedom robotic patient table called the RoboCouch, and an X-ray imaging system that can take real-time images in two orthogonal orientations simultaneously $[79,80]$. The two simultaneous, intraoperative X-ray images are not sufficient to provide good definition of the tumor, but are used to register a high-definition preoperative CT image. The robotic arm can then provide the preplanned radiation dosage with a wide range of orientations. For targets that move during treatment (e.g., due to breathing), the optional synchrony system can optically track the tissue surface, correlate the motion of the tissue surface to the motion of radio-opaque fiducials inserted near the target, and thus continuously predict target motion [81]. The intraoperative tracking obviates the need for a stereotactic frame, reducing patient trauma and making it practical to fractionate the dosage over longer time periods.

The Novalis with TrueBeam STx (BrainLab Inc. and Varian Medical Systems, previously Novalis and Trilogy, initial FDA clearance 2000, Figure 5(b)) is also a frameless system with a linear accelerator, but with micro-multileaf collimators for beam shaping [82-84]. Similar to CyberKnife, intraoperative X-rays are compared with a CT, and skinmounted fiducials are optically tracked in real-time. The delivery system also includes cone beam CT. The patient is moved into position on top of a six degree of freedom robotic couch. The major differences between Cyberknife and Novalis are that the Cyberknife radiation source has more degrees of freedom to be oriented around the patient while the Novalis can shape the radiation beam and claim reduced out-of-field dosage $[85,86]$.

\section{Emergency Response}

Few medical robot systems are suitable for use outside of the operating room, despite significant research funding on medical devices for disaster response and battlefield medicine. Typical goals for such research include improved extraction of patients from dangerous environments, rapid diagnosis of injuries, and semiautonomous delivery of lifesaving interventions. Current Emergency Response robots are little more than single-motor systems, but those systems can be controlled by health monitors to minimize the necessary attention by Emergency Responders. Such a feedback control makes it more likely that such systems will be autonomous, for example, automated external defibrillators.

The AutoPulse Plus (ZOLL Medical Corp., previously by Revivant) is an automated, portable device that combines the functions of the AutoPulse (FDA clearance 2008, Figure 6(a)) cardiopulmonary resuscitation device and the E Series monitor/defibrillator (FDA clearance 2010) [87, 88]. Consisting of a half-backboard containing a battery-powered motor that actuates a chest band, the AutoPulse rhythmically tightens 


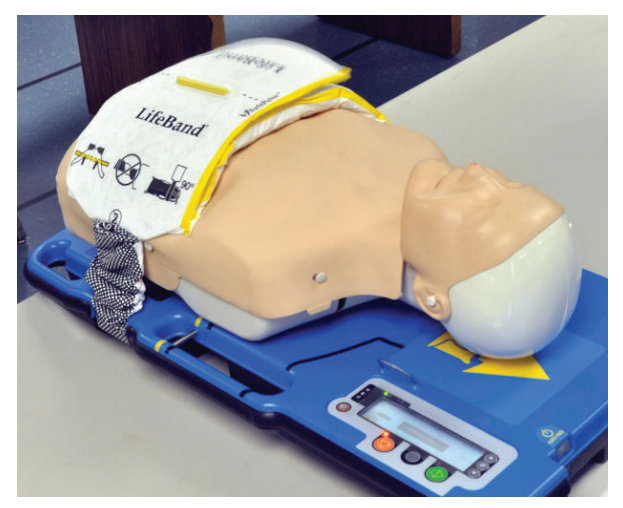

(a) AutoPulse by ZOLL Medical Corp.

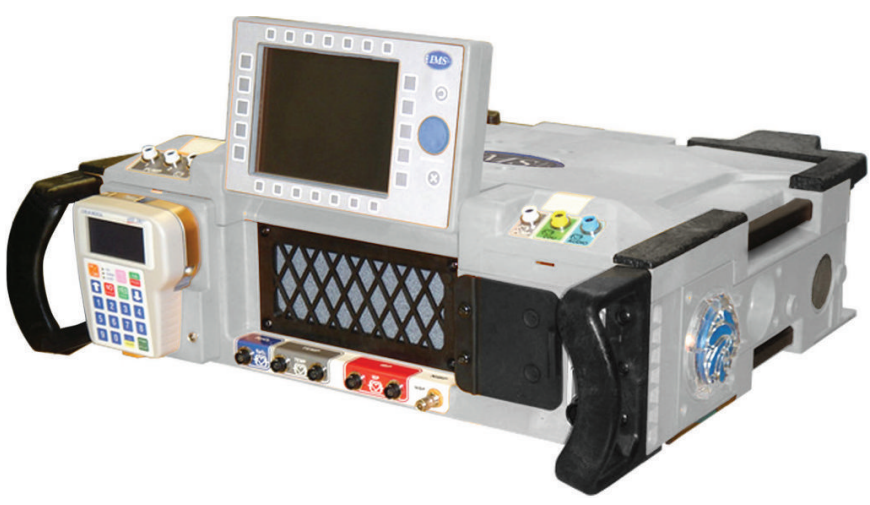

(b) LS-1 by Integrated Medical Systems Inc.

Figure 6: Commercially available Emergency Response robots perform simple actuations compared to robots in other medical disciplines, but their actions are tightly coupled with patient measurements. The AutoPulse image is (C2012 ZOLL Medical Corp. The LS-1 image is (C)2012 Integrated Medical Systems Inc.

the band to perform chest compressions. The tightness of the band during compressions is a function of the patient's resting chest size, to adjust for interpatient variability. Meanwhile, the E Series monitor/defibrillator measures the rate and depth of chest compressions in real time and filters cardiopulmonary resuscitation artifacts from the electrocardiogram signal. If combined with an automatic batterypowered ventilator, for example, the SAVe (AutoMedx Inc., FDA clearance 2007), basic cardiopulmonary emergency response treatments could be automated while on battery power.

The LS-1 "suitcase intensive care unit" (Integrated Medical Systems Inc., previous generation called MedEx 1000 , previous generation called LSTAT, FDA clearance 2008, Figure 6(b)) takes an inclusive approach to portable life support [89]. The system contains a ventilator with oxygen and carbon dioxide monitoring, electrocardiogram, invasive and noninvasive blood pressure monitoring, fluid/drug infusion pumps, temperature sensing, and blood oxygen level measurement. The LS- 1 is battery powered and can be powered by facility or vehicular electrical sources. The system is FDA-cleared for remote control of its diagnostic and therapeutic capabilities.

\section{Prosthetics and Exoskeletons}

Microprocessor-controlled prosthetics have been available since 1993, specifically the Intelligent Prosthesis knee (Chas. A. Blatchford \& Sons, Ltd.). Several microprocessorcontrolled prosthetics exist today, predominantly for knee prosthetics, hand prosthetics, and exoskeletons. For example, one current generation knee prosthetic is the C-leg (Otto Bock, FDA clearance 1999, CE mark) which is designed to automatically adjust the swing phase dynamics and improve stability during the stance phase by controlling knee flexion [90]. An example of hand prosthetic is the i-limb ultrahand (Touch Bionics, previous version i-limb hand, FDA clearance and CE mark), the first commercially available hand prosthesis with five individually powered digits, controlled via myoelectric signals generated by muscles in the remaining portion of the patient's limb [91]. For wheelchair users, the ReWalk (Argo Medical Technologies, FDA clearance 2011, CE mark 2010) is one walking assistance exoskeleton that allows users to stand, walk, and climb stairs and is controlled with a wrist-mounted remote and a posture detection sensor [92]. Significant research on exoskeletons is ongoing, such as the research on upper-limb exoskeletons by Rosen and Perry [93]. For further information in the area of prosthetics and exoskeletons see the works by Kazerooni [94] and Bogue [95].

\section{Assistive and Rehabilitation Systems}

Assistive robotic systems are designed to allow people with disabilities more autonomy, and they cover a wide range of everyday tasks. In 1992, Handy 1 (Rehab Robotics, Ltd.) became the first commercial assistive robot [96]; it interacts with different trays for tasks such as eating, shaving, and painting, and it is controlled by a single switch input to select the desired action. One task-specific system is the Neater Eater (Neater Solutions Ltd.), a modular device that scoops food from a plate to a person's mouth, and can be controlled manually or via head or foot switches. More general systems rely on arms with many degrees of freedom, such as Exact Dynamics' iARM, a robotic arm with a two-fingered grasper, that attaches to electric wheelchairs and can be controlled via keypad, joystick, or single button.

Rehabilitation systems can be similar to assistive systems, but are designed to facilitate recovery by delivering therapy and measuring the patient's progress, often following a stroke [97]. The Mobility System (Myomo, Inc.) is a wearable robotic device that moves the patient's arm in response to his/her muscle signals, thus creating feedback to facilitate muscle reeducation. The InMotion (Interactive Motion Technologies, based on the MIT-MANUS research platform) is a robotic arm that moves, guides, or perturbs the patient's arm within a planar workspace, while recording motions, 
velocities, and forces to evaluate progress [98]. For information on research efforts, see Dallaway et al. [99] for an overview of thirty assistive and rehabilitation systems, such as the MASTER II system that uses a rail-mounted robotic arm to make manually controlled, remote-controlled, or preprogrammed motions for various domestic and office tasks [100]. Difficulties in developing rehabilitation robots and potential future uses are investigated by Ceccarelli [101].

\section{Current Research and Development in Medical Robotics}

Many more medical robots are currently being researched $[16,19,21]$. Such research will lead to the new capabilities of future commercial systems. This section discusses just a few systems of note.

11.1. RAVEN and MiroSurge. Two prominent academic robot-assisted surgical systems are currently used for research into endoscopic telesurgery: RAVEN II and MiroSurge. The RAVEN II (University of Washington and UC Santa Cruz) is a teleoperated laparoscopic system that was designed to maximize surgical performance based on objective clinical measurements [102-104]. The system has two patient-side arms that are cable-driven with 7 degrees of freedom each. The arm kinematics are based on a spherical mechanism such that the tool always passes through a remote center (e.g., the insertion point for minimally invasive surgery). The length and angles of the links were optimized to maximize performance throughout the workspace. The arms are lighter, smaller, and less expensive than current robotic systems for laparoscopy. The instrument controllers are haptic devices, allowing force feedback on the operator's hands based on tool forces or virtual fixtures (e.g., forbidden regions) defined with respect to patient anatomy (see [105-107] for the impact of haptics on surgery). Teleoperation experiments have been conducted with the RAVEN, including routing the data transmission through an unmanned aircraft. In February 2012, five systems were provided to various other surgical robotics research labs to spur collaboration and further development efforts.

In another endoscopic research effort, the German Aerospace Center (DLR) is developing MiroSurge to be highly versatile with respect to the number of surgical domains, arm-mounting locations, number of robots, different control modes (e.g., control of position versus control of force), and the ability to integrate with other technologies [108]. The expectation is for a base robot system to hold specialized instruments, such as DLR's MICA instrument (which is itself a robotic tool with 3 degrees of freedom and force sensing) [109]. By using a general robotic base to hold a specialized robotic instrument that has its own motors, sensors, and control electronics, the same base system can be specialized for various procedures just by switching the instrument. The base robot, the DLR Miro, masses $10 \mathrm{~kg}$ with a $3 \mathrm{~kg}$ payload and has serial kinematics that resemble the kinematics of the human arm, with joint ranges and link lengths optimized based on certain medical procedures
[110]. Unlike the RAVEN II, the MIRO arm does not have a remote center of motion, and thus must be controlled to direct the instrument through any insertion point, but is more easily able to handle moving insertion points (e.g., through the chest wall during respiration).

11.2. Amadeus. Titan Medical Inc. is currently developing Amadeus, a four-armed laparoscopic surgical robot system, to compete with Intuitive Surgical's da Vinci system. The Amadeus uses snakelike multiarticulating arms for improved maneuverability, and the system is being designed to facilitate teleoperation for long-distance surgery. Human trials are planned for late 2013.

11.3. NeuroArm and MrBot. At least two renowned research systems are investigating improved MR-compatible robots. The neuroArm (University of Calgary, MacDonald Dettwiler and Associates, IMRIS) is a two-armed, MRI-guided neurosurgical robot actuated via piezoelectric motors [111, 112]. The neuroArm end effectors are equipped with 3 degrees of freedom optical force sensors and are accurate to tens of micrometers. The MrBot (Johns Hopkins University) is a parallel linkage arm designed for MRI-guided access of the prostate gland, actuated by novel pneumatic stepper motors for reduced MR interference [113].

11.4. TraumaPod. TraumaPod (highly collaborative, led by SRI International) is a semi-autonomous telerobotic surgical system designed to be rapidly deployable [114]. The surgical cell consists of a surgical robot (da Vinci for Phase I testing), Scrub Nurse Subsystem, Tool Rack System, Supply Dispensing System, Patient Imaging System (a movable Xray tube), predecessor of the aforementioned LS-1 ("suitcase intensive care unit”), and Supervisory Controller System. The TraumaPod has demonstrated successful teleoperation of a bowel closure and shunt placement on a phantom without a human in the surgical cell. That success implies the potential for increased automation in the operating room, though challenges were reported in sterilization, anesthesia, and robustness.

11.5. HeartLander. The heart has long been a target for surgical robots and various systems continue to investigate how best to treat cardiac diseases, particularly while the heart is beating (e.g., see Section 6) [115]. The HeartLander (HeartLander Surgical) is a minimally invasive robot that uses suction to crawl around the surface of the heart $[116,117]$. The system is designed for intrapericardial drug delivery, cell transplantation, epicardial atrial ablation, and other such procedures.

11.6. Robots In Vivo. Various groups are expanding and exploring the da Vinci system's approach to enhance surgery by increasing the dexterity of the tool inside the patient. One such example is the University of Nebraska's laparoscopic robotic system for research into single-site surgeries [118]. The system has two arms with six degrees of freedom each, and those arms are fully inserted into the abdomen. 
The expectation is that, by increasing the tool's dexterity inside the patient, fewer incisions will be needed to insert instruments because multiple tools/arms can pass through a single incision and then spread out inside the patient. Further, miniaturizing the robotics reduces the difficulty in working with (and around) the system in the operating room. A limited number of animal trials (colon resections) have been performed to demonstrate feasibility.

Swallowable capsules take patient trauma reduction to an extreme, but current systems are limited to diagnostic uses. Core temperature measurement has been FDA cleared since 1990, by CorTemp (HQ Inc., formerly HTI Technologies). More recently, capsule endoscopy systems (PillCam by Given Imaging with FDA clearance 2001, and EndoCapsule by Olympus with FDA clearance 2007) consist of a forwardlooking wide-angle camera taking regularly timed pictures, a battery, and lights, all contained in a capsule [119, 120]. SmartPill (SmartPill Corp., FDA clearance 2006) utilizes multiple sensors to measure pressure, $\mathrm{pH}$ level, gastric emptying time, and bowel emptying time [121]. Sayaka (RF Co Ltd.) is a novel design, not FDA cleared, using a lateral-facing camera that rotates inside the capsule to image the entire tract and is designed without a battery, instead relying on an externally applied magnetic field for inductive power supply [122]. For the future, many enhancements have been proposed, including biopsy, real-time localization of the capsule, drug delivery, ultrasonic imaging, increasing motility by electrically inducing peristalsis, and utilizing an active locomotion system involving treads or legs [123].

In a more dramatic approach to in vivo robotics, micro/ nanotechnology is a multibillion dollar area of research [124, 125], including investigation for various medical robotic uses such as inexpensive directable drug delivery vehicles, radio-controlled biomolecules, tissue micromanipulation platforms, artificial mechanical white blood cells, and many other therapeutic approaches that may benefit from robots working at the cellular level [126-129]. Construction of functional systems is an ongoing area of research, particularly with respect to generating and powering motion. Many current prototypes are propelled and guided via magnetic fields, though some utilize external electrical energy sources $[130,131]$. To the author's knowledge, clinical trials have not begun for any medical micro/nanorobot.

\section{Discussion}

Medical robotics is a young and relatively unexplored field made possible by technical improvements over the past couple of decades. Currently available systems have been available for too short time to allow long-term studies. Nor are the benefits potentially provided by medical robots fully understood. Medical robots have only passed through a few technological generations and the technology continues to change and leap into new areas. Yet by looking at the current market and representative research systems, educated guesses can be made about the impacts of robots on near-future medicine.
In surgical robotics, there has been a trend away from autonomous or even semiautonomous motions, and toward synergistic manipulation and virtual fixtures. Thus, the robot acts as a guidance tool, providing information (and possibly a physical nudge) to keep the surgeon on target. Such use requires accurate localization of the tissues in the surgical site, even as the tissues are manipulated during surgery. Improved imaging systems (e.g., Explorer, an intraoperative soft tissue tracker by Pathfinder Therapeutics [132]) or robot compatibility with MRI or CT will provide that localization. In particular, MRI-guided robots will benefit from intraoperative $3 \mathrm{D}$ images with excellent soft tissue contrast and accurate registration between the tool and the tissue, thus allowing precise virtual fixtures, "snapto" and "stand-off" behaviors. Further, such imaging will allow modeling and rapid prototyping of patient-specific templates/jigs/implants.

The physical designs for medical robots will continue to improve, reducing expense and size, while minimizing or compensating for nonidealities such as flexion, for example, the CRIGOS robot [133]. With better physical designs, semiautonomous behavior will likely become more useful. "Macros" may become commonplace, wherein the surgeon presses a button and the robot performs a preprogrammed motion, such as passing a suture needle between graspers, or the Sensei's autoretract feature [13].

Robots will see more use for medical training purposes, bolstered by improved tissue-modeling capabilities, by the increasing objectivity in healthcare assessment, by advances in computer simulations, and as a result of increased data mining arising naturally from improved data connectivity between devices and between institutions. Some such systems are already available, such as the aforementioned da Vinci Skills Simulator, the Virtual I.V. Simulator by Laerdal, and the EndoscopyVR Surgical Simulator by CAE. For the same reasons, robotics will continue to make possible new medical procedures and treatments, such as new Single-Port Access procedures.

Even as robots are developed for new medical areas, other tools may encroach on medical needs currently filled by robots. Medical robots must develop a firm basis in improved medical outcomes, or risk being displaced by pharmaceuticals, tissue engineering, gene therapy, and rapid innovation in manual tools (e.g., the SPIDER Surgical System by TransEnterix, and the EndoStitch by Covidien). To that end, improvements in medical robotics must address and solve real problems in healthcare, ultimately providing a clear improvement in quality of life when compared with the alternatives.

\section{References}

[1] Intuitive Surgical Incorporation, "Webpage on da Vinci clinical evidence," March 2012, http://www.intuitivesurgical .com/company/clinical-evidence.

[2] Intuitive Surgical Incorporation, "Webpage on da Vinci regulatory approval," March 2012, http://www.intuitivesurgical .com/company/regulatory-clearance.html. 
[3] H. Lavery, D. Samadi, and A. Levinson, "Not a zerosum game: the adoption of robotics has increased overall prostatectomy utilization in the united states," in Proceedings of the American Urological Association Annual Meeting, Poster Session, Washington, DC, USA, 2011.

[4] R. D. Howe and Y. Matsuoka, "Robotics for surgery," Annual Review of Biomedical Engineering, vol. 1, pp. 211-240, 1999.

[5] B. Davies, "A review of robotics in surgery," Proceedings of the Institution of Mechanical Engineers H, vol. 214, no. 1, pp. 129-140, 2000.

[6] R. H. Taylor and D. Stoianovici, "Medical robotics in computer-integrated surgery," IEEE Transactions on Robotics and Automation, vol. 19, no. 5, pp. 765-781, 2003.

[7] A. R. Lanfranco, A. E. Castellanos, J. P. Desai, and W. C. Meyers, "Robotic surgery: a current perspective," Annals of Surgery, vol. 239, no. 1, pp. 14-21, 2004.

[8] P. Berkelman, J. Troccaz, and P. Cinquin, "Body-supported medical robots: a survey," Journal of Robotics and Mechatronics, vol. 16, pp. 513-519, 2004.

[9] L. Guo, X. Pan, Q. Li, F. Zheng, and Z. Bao, "A survey on the gastrointestinal capsule micro-robot based on wireless and optoelectronic technology," Journal of Nanoelectronics and Optoelectronics, vol. 7, no. 2, pp. 123-127, 2012.

[10] C. Stüer, F. Ringel, M. Stoffel, A. Reinke, M. Behr, and B. Meyer, "Robotic technology in spine surgery: current applications and future developments," Intraoperative Imaging, vol. 109, pp. 241-245, 2011.

[11] S. Badaan and D. Stoianovici, "Robotic systems: past, present, and future," in Robotics in Genitourinary Surgery, pp. 655665, Springer, New York, NY, USA, 2011.

[12] I. Singh, "Robotics in urological surgery: review of current status and maneuverability, and comparison of robotassisted and traditional laparoscopy," Computer Aided Surgery, vol. 16, no. 1, pp. 38-45, 2011.

[13] G. P. Moustris, S. C. Hiridis, K. M. Deliparaschos, and K. M. Konstantinidis, "Evolution of autonomous and semiautonomous robotic surgical systems: a review of the literature," International Journal of Medical Robotics and Computer Assisted Surgery, vol. 7, no. 4, pp. 375-392, 2011.

[14] B. Challacombe and D. Stoianovici, "The basic science of robotic surgery," in Urologic Robotic Surgery in Clinical Practice, pp. 1-23, 2009.

[15] H. Kenngott, L. Fischer, F. Nickel, J. Rom, J. Rassweiler, and B. Muller-Stich, "Status of robotic assistance: a less traumatic and more accurate minimally invasive surgery?" Langenbeck's Archives of Surgery, vol. 397, no. 3, pp. 1-9, 2012.

[16] P. Gomes, "Surgical robotics: reviewing the past, analysing the present, imagining the future," Robotics and ComputerIntegrated Manufacturing, vol. 27, no. 2, pp. 261-266, 2011.

[17] A. M. Okamura, M. J. Matarić, and H. I. Christensen, "Medical and health-care robotics," IEEE Robotics and Automation Magazine, vol. 17, no. 3, pp. 26-37, 2010.

[18] S. Najarian, M. Fallahnezhad, and E. Afshari, "Advances in medical robotic systems with specific applications in surgery-a review," Journal of Medical Engineering and Technology, vol. 35, no. 1, pp. 19-33, 2011.

[19] J. Rosen, B. Hannaford, and R. Satava, Eds., Surgical Robotics: Systems Applications and Visions, Springer, New York, NY, USA, 2011.

[20] N. Nathoo, M. C. Çavuşoğlu, M. A. Vogelbaum, and G. H. Barnett, "In touch with robotics: neurosurgery for the future," Neurosurgery, vol. 56, no. 3, pp. 421-431, 2005.

[21] T. Haidegger, L. Kovacs, G. Fordos, Z. Benyo, and P. Kazanzides, "Future trends in robotic neurosurgery," in
Proceedings of the 14th Nordic-Baltic Conference on Biomedical Engineering and Medical Physics (NBC '08), pp. 229-233, Springer, June 2008.

[22] Y. S. Kwoh, J. Hou, E. A. Jonckheere, and S. Hayati, "A robot with improved absolute positioning accuracy for CT guided stereotactic brain surgery," IEEE Transactions on Biomedical Engineering, vol. 35, no. 2, pp. 153-160, 1988.

[23] D. Glauser, H. Fankhauser, M. Epitaux, J. L. Hefti, and A. Jaccottet, "Neurosurgical robot Minerva: first results and current developments," Journal of Image Guided Surgery, vol. 1, no. 5, pp. 266-272, 1995.

[24] T. R. K. Varma and P. Eldridge, "Use of the NeuroMate stereotactic robot in a frameless mode for functional neurosurgery," International Journal of Medical Robotics and Computer Assisted Surgery, vol. 2, no. 2, pp. 107-113, 2006.

[25] Q. H. Li, L. Zamorano, A. Pandya, R. Perez, J. Gong, and F. Diaz, "The application accuracy of the NeuroMate robota quantitative comparison with frameless and frame-based surgical localization systems," Computer Aided Surgery, vol. 7, no. 2, pp. 90-98, 2002.

[26] P. Morgan, T. Carter, S. Davis et al., "The application accuracy of the pathfinder neurosurgical robot," in International Congress Series, vol. 1256, pp. 561-567, Elsevier, Amsterdam, The Netherlands, 2003.

[27] G. Deacon, A. Harwood, J. Holdback et al., "The pathfinder image-guided surgical robot," Proceedings of the Institution of Mechanical Engineers H, vol. 224, no. 5, pp. 691-713, 2010.

[28] J. Brodie and S. Eljamel, "Evaluation of a neurosurgical robotic system to make accurate burr holes," International Journal of Medical Robotics and Computer Assisted Surgery, vol. 7, no. 1, pp. 101-106, 2011.

[29] L. Joskowicz, R. Shamir, Z. Israel, Y. Shoshan, and M. Shoham, "Renaissance robotic system for keyhole cranial neurosurgery: in-vitro accuracy study," in Proceedings of the Simposio Mexicano en Ciruga Asistida por Computadora y Procesamiento de Imgenes Mdicas (MexCAS '11), 2011.

[30] D. P. Devito, L. Kaplan, R. Dietl et al., "Clinical acceptance and accuracy assessment of spinal implants guided with spineassist surgical robot: retrospective study," Spine, vol. 35, no. 24, pp. 2109-2115, 2010.

[31] M. Yang, J. Jung, J. Kim et al., "Current and future of spinal robot surgery," Korean Journal of Spine, vol. 7, no. 2, pp. 6165, 2010.

[32] J. E. Lang, S. Mannava, A. J. Floyd et al., "Robotic systems in orthopaedic surgery," Journal of Bone and Joint Surgery B, vol. 93, no. 10, pp. 1296-1299, 2011.

[33] W. L. Bargar, A. Bauer, and M. Börner, "Primary and revision total hip replacement using the robodoc system," Clinical Orthopaedics and Related Research, vol. 354, pp. 82-91, 1998.

[34] A. P. Schulz, K. Seide, C. Queitsch et al., "Results of total hip replacement using the Robodoc surgical assistant system: clinical outcome and evaluation of complications for 97 procedures," International Journal of Medical Robotics and Computer Assisted Surgery, vol. 3, no. 4, pp. 301-306, 2007.

[35] P. Kazanzides, J. Zuhars, B. Mittelstadt, and R. H. Taylor, "Force sensing and control for a surgical robot," in Proceedings of the IEEE International Conference on Robotics and Automation, pp. 612-617, May 1992.

[36] A. D. Pearle, P. F. O'Loughlin, and D. O. Kendoff, "Robotassisted unicompartmental knee arthroplasty," Journal of Arthroplasty, vol. 25, no. 2, pp. 230-237, 2010.

[37] A. D. Pearle, D. Kendoff, V. Stueber, V. Musahl, and J. A. Repicci, "Perioperative management of unicompartmental knee arthroplasty using the MAKO robotic arm system 
(MAKOplasty)," American Journal of Orthopedics, vol. 38, no. 2, pp. 16-19, 2009.

[38] C. Plaskos, P. Cinquin, S. Lavallée, and A. J. Hodgson, "Praxiteles: a miniature bone-mounted robot for minimal access total knee arthroplasty," The International Journal of Medical Robotics and Computer Assisted Surgery, vol. 1, no. 4, pp. 67-79, 2005.

[39] D. Koulalis, P. F. O’Loughlin, C. Plaskos, D. Kendoff, M. B. Cross, and A. D. Pearle, "Sequential versus automated cutting guides in computer-assisted total knee arthroplasty," Knee, vol. 18, no. 6, pp. 436-442, 2010.

[40] G. Brisson, T. Kanade, A. DiGioia, and B. Jaramaz, "Precision freehand sculpting of bone," in Proceedings of the 7th International Conference on Medical Image Computing and Computer-Assisted Intervention (MICCAI '04), pp. 105-112, September 2004.

[41] G. Brisson, The Precision Freehand Sculptor: a Robotic Tool for Less Invasive Joint Replacement Surgery, ProQuest, 2008.

[42] P. L. Yen and B. L. Davies, "Active constraint control for image-guided robotic surgery," Proceedings of the Institution of Mechanical Engineers H, vol. 224, no. 5, pp. 623-631, 2010.

[43] A. G. Harrell and B. T. Heniford, "Minimally invasive abdominal surgery: lux et veritas past, present, and future," American Journal of Surgery, vol. 190, no. 2, pp. 239-243, 2005.

[44] G. Dogangil, B. L. Davies, and F. Rodriguez Y Baena, "A review of medical robotics for minimally invasive soft tissue surgery," Proceedings of the Institution of Mechanical Engineers $H$, vol. 224, no. 5, pp. 653-679, 2010.

[45] C. Kuo and J. Dai, "Robotics for minimally invasive surgery: a historical review from the perspective of kinematics," in Proceedings of the International Symposium on History of Machines and Mechanisms, pp. 337-354, Springer, 2009.

[46] S. J. Harris, F. Arambula-Cosio, and Q. Mei, "The probotan active robot for prostate resection," Proceedings of the Institution of Mechanical Engineers H, vol. 211, no. 4, pp. 317325, 1997.

[47] G. H. Ballantyne, "Robotic surgery, telerobotic surgery, telepresence, and telementoring: review of early clinical results," Surgical Endoscopy and Other Interventional Techniques, vol. 16, no. 10, pp. 1389-1402, 2002.

[48] G. T. Sung and I. S. Gill, "Robotic laparoscopic surgery: a comparison of the da Vinci and Zeus systems," Urology, vol. 58, no. 6, pp. 893-898, 2001.

[49] J. Marescaux, J. Leroy, M. Gagner et al., "Transatlantic robotassisted telesurgery,” Nature, vol. 413, no. 6854, pp. 379-380, 2001.

[50] P. Mozer, J. Troccaz, and D. Stoinaovici, "Robotics in urology: past, present, and future," in Atlas of Robotic Urologic Surgery, L. Su, Ed., Current Clinical Urology, ch. 1, pp. 3-13, Springer, New York, NY, USA, 2011.

[51] K. Shah and R. Abaza, "Comparison of intraoperative outcomes using the new and old generation da Vinci robot for robot-assisted laparoscopic prostatectomy," British Journal of Urology International, vol. 108, no. 10, pp. 1642-1645, 2011.

[52] J. Bodner, H. Wykypiel, G. Wetscher, and T. Schmid, "First experiences with the da Vinci operating robot in thoracic surgery," European Journal of Cardio-Thoracic Surgery, vol. 25, no. 5, pp. 844-851, 2004.

[53] A. Tewari, A. Srivasatava, and M. Menon, "A prospective comparison of radical retropubic and robot-assisted prostatectomy: experience in one institution," British Journal of Urology International, vol. 92, no. 3, pp. 205-210, 2003.
[54] S. Maeso, M. Reza, J. A. Mayol et al., "Efficacy of the da Vinci surgical system in abdominal surgery compared with that of laparoscopy: a systematic review and meta-analysis," Annals of Surgery, vol. 252, no. 2, pp. 254-262, 2010.

[55] R. E. Link, S. B. Bhayani, and L. R. Kavoussi, "A prospective comparison of robotic and laparoscopic pyeloplasty," Annals of Surgery, vol. 243, no. 4, pp. 486-491, 2006.

[56] A. Amodeo, A. Linares Quevedo, J. V. Joseph, E. Belgrano, and H. R. H. Patel, "Robotic laparoscopic surgery: cost and training," Minerva Urologica e Nefrologica, vol. 61, no. 2, pp. 121-128, 2009.

[57] W. Jeong, F. Petros, and C. Rogers, Robotic Surgery: Basic Instrumentation and Troubleshooting, ch. 72, WileyBlackwell, Hoboken, NJ, USA, 2012.

[58] M. A. Lerner, M. Ayalew, W. J. Peine, and C. P. Sundaram, "Does training on a virtual reality robotic simulator improve performance on the da Vinci surgical system?" Journal of Endourology, vol. 24, no. 3, pp. 467-472, 2010.

[59] K. Cleary and T. M. Peters, "Image-guided interventions: technology review and clinical applications," Annual Review of Biomedical Engineering, vol. 12, pp. 119-142, 2010.

[60] M. E. Hagen, O. J. Wagner, I. Inan et al., "Robotic singleincision transabdominal and transvaginal surgery: initial experience with intersecting robotic arms," International Journal of Medical Robotics and Computer Assisted Surgery, vol. 6, no. 3, pp. 251-255, 2010.

[61] M. Kroh, K. El-Hayek, S. Rosenblatt et al., "First human surgery with a novel single-port robotic system: cholecystectomy using the da Vinci Single-Site platform," Surgical Endoscopy, vol. 25, no. 11, pp. 3566-3573, 2011.

[62] M. Stark, T. Benhidjeb, S. Gidaro, and E. Morales, "The future of telesurgery: a universal system with haptic sensation," Journal of the Turkish-German Gynecological Association, vol. 13, no. 1, pp. 74-76, 2012.

[63] S. DiMaio and S. Salcudean, "Needle steering and modelbased trajectory planning," in Proceedings of the 6th International Conference on Medical Image Computing and Computer-Assisted Intervention (MICCAI '03), pp. 33-40, 2003.

[64] H. Delingette, "Toward realistic soft-tissue modeling in medical simulation," Proceedings of the IEEE, vol. 86, no. 3, pp. 512-523, 1998.

[65] A. Melzer, B. Gutmann, T. Remmele et al., "Innomotion for percutaneous image-guided interventions," IEEE Engineering in Medicine and Biology Magazine, vol. 27, no. 3, pp. 66-73, 2008.

[66] M. Li, A. Kapoor, D. Mazilu, and K. A. Horvath, "Pneumatic actuated robotic assistant system for aortic valve replacement under MRI guidance," IEEE Transactions on Biomedical Engineering, vol. 58, no. 2, pp. 443-451, 2011.

[67] S. Zangos, A. Melzer, K. Eichler et al., "MR-compatible assistance system for biopsy in a high-field-strength system: initial results in patients with suspicious prostate lesions," Radiology, vol. 259, no. 3, pp. 903-910, 2011.

[68] H. J. Swan, W. Ganz, J. Forrester, H. Marcus, G. Diamond, and D. Chonette, "Catheterization of the heart in man with use of a flow-directed balloon-tipped catheter," The New England Journal of Medicine, vol. 283, no. 9, pp. 447-451, 1970.

[69] M. R. Franz, D. Burkhoff, and H. Spurgeon, "In vitro validation of a new cardiac catheter technique for recording monophasic action potentials," European Heart Journal, vol. 7, no. 1, pp. 34-41, 1986. 
[70] J. M. Gore, R. J. Goldberg, D. H. Spodick, J. S. Alpert, and J. E. Dalen, "A community-wide assessment of the use of pulmonary artery catheters in patients with acute myocardial infarction," Chest, vol. 92, no. 4, pp. 721-727, 1987.

[71] D. Steven, H. Servatius, T. Rostock et al., "Reduced fluoroscopy during atrial fibrillation ablation: benefits of robotic guided navigation," Journal of Cardiovascular Electrophysiology, vol. 21, no. 1, pp. 6-12, 2010.

[72] V. Y. Reddy, P. Neuzil, Z. J. Malchano et al., "Viewsynchronized robotic image-guided therapy for atrial fibrillation ablation: experimental validation and clinical feasibility," Circulation, vol. 115, no. 21, pp. 2705-2714, 2007.

[73] K. R. J. Chun, B. Schmidt, B. Köktürk et al., "Catheter ablation-new developments in robotics," Herz, vol. 33, no. 8, pp. 586-589, 2008.

[74] C. V. Riga, C. D. Bicknell, D. Wallace, M. Hamady, and N. Cheshire, "Robot-assisted antegrade in-situ fenestrated stent grafting," CardioVascular and Interventional Radiology, vol. 32, no. 3, pp. 522-524, 2009.

[75] S. Ernst, F. Ouyang, C. Linder et al., "Initial experience with remote catheter ablation using a novel magnetic navigation system," Circulation, vol. 109, no. 12, pp. 1472-1475, 2004.

[76] J. K. R. Chun, S. Ernst, S. Matthews et al., "Remote-controlled catheter ablation of accessory pathways: results from the magnetic laboratory," European Heart Journal, vol. 28, no. 2, pp. 190-195, 2007.

[77] L. Leksell, "Stereotactic radiosurgery," Journal of Neurology Neurosurgery and Psychiatry, vol. 46, no. 9, pp. 797-803, 1983.

[78] R. Schulz and N. Agazaryan, Shaped-Beam Radiosurgery: State of the Art, Springer, New York, NY, USA, 2011.

[79] J. R. Adler Jr., S. D. Chang, M. J. Murphy, J. Doty, P. Geis, and S. L. Hancock, "The cyberknife: a frameless robotic system for radiosurgery," Stereotactic and Functional Neurosurgery, vol. 69, no. 1-4, pp. 124-128, 1997.

[80] G. J. Gagnon, N. M. Nasr, J. J. Liao et al., "Treatment of spinal tumors using cyberKnife fractionated stereotactic radiosurgery: pain and quality-of-life assessment after treatment in 200 patients," Neurosurgery, vol. 64, no. 2, pp. 297306, 2009.

[81] M. Hoogeman, J. B. Prevost, J. Nuyttens, J. Poll, P. Levendag, and B. Heijmen, "Clinical accuracy of the respiratory tumor tracking system of the cyberknife: assessment by analysis of $\log$ files," International Journal of Radiation Oncology *Biology* Physics, vol. 74, no. 1, pp. 297-303, 2009.

[82] J. P. Rock, S. Ryu, F. F. Yin, F. Schreiber, and M. Abdulhak, "The evolving role of stereotactic radiosurgery and stereotactic radiation therapy for patients with spine tumors," Journal of Neuro-Oncology, vol. 69, no. 1-3, pp. 319-334, 2004.

[83] R. E. Wurm, S. Erbel, I. Schwenkert et al., "Novalis frameless image-guided noninvasive radiosurgery: initial experience," Neurosurgery, vol. 62, no. 5, pp. A11-A17, 2008.

[84] Z. Chang, T. Liu, J. Cai, Q. Chen, Z. Wang, and F. Yin, "Evaluation of integrated respiratory gating systems on a novalis tx system," Journal of Applied Clinical Medical Physics, vol. 12, no. 3, article 3495, 2011.

[85] A. Liu, N. Agazaryan, C. Yu, H. Han, T. Schultheiss, and J. Wong, "A multi-center consortium study of competing platforms for intracranial stereotactic irradiation," International Journal of Radiation Oncology * Biology* Physics, vol. 72, supplement 1, pp. S213-S213, 2008.

[86] M. Abacioglu, "Advances in technology in radiation oncology," Oncology, vol. 2, no. 1, pp. 11-14, 2012.
[87] H. R. Halperin, N. Paradis, J. P. Ornato et al., "Cardiopulmonary resuscitation with a novel chest compression device in a porcine model of cardiac arrest: improved hemodynamics and mechanisms," Journal of the American College of Cardiology, vol. 44, no. 11, pp. 2214-2220, 2004.

[88] A. Hallstrom, T. D. Rea, M. R. Sayre et al., "Manual chest compression vs use of an automated chest compression device during resuscitation following out-of-hospital cardiac arrest: a randomized trial," Journal of the American Medical Association, vol. 295, no. 22, pp. 2620-2628, 2006.

[89] R. Palmer, "Integrated diagnostic and treatment devices for enroute critical care of patients within theater," in Proceedings of the RTO Human Factors and Medicine Panel Symposium, Amsterdam, The Netherlands, October 2010.

[90] R. Seymour, B. Engbretson, K. Kott et al., "Comparison between the C-leg microprocessor-controlled prosthetic knee and non-microprocessor control prosthetic knees: a preliminary study of energy expenditure, obstacle course performance, and quality of life survey," Prosthetics and Orthotics International, vol. 31, no. 1, pp. 51-61, 2007.

[91] O. Otr, H. A. Reinders-Messelink, R. M. Bongers, H. Bouwsema, and C. K. Van Der Sluis, "The i-LIMB hand and the DMC plus hand compared: a case report," Prosthetics and Orthotics International, vol. 34, no. 2, pp. 216-220, 2010.

[92] K. Low, "Robot-assisted gait rehabilitation: from exoskeletons to gait systems," in Proceedings of the Defense Science Research Conference and Expo (DSR '11), pp. 1-10, August 2011.

[93] J. Rosen and J. C. Perry, "Upper limb powered exoskeleton," International Journal of Humanoid Robotics, vol. 4, no. 3, pp. 529-548, 2007.

[94] H. Kazerooni, "Exoskeletons for human performance augmentation," in Springer Handbook of Robotics, B. Siciliano and O. Khatib, Eds., Springer, New York, NY, USA, 2008.

[95] R. Bogue, "Exoskeletons and robotic prosthetics: a review of recent developments," Industrial Robot, vol. 36, no. 5, pp. 421-427, 2009.

[96] M. J. Topping and J. K. Smith, "The development of Handy 1. A robotic system to assist the severely disabled," Technology and Disability, vol. 10, no. 2, pp. 95-105, 1999.

[97] M. Hillman, "Rehabilitation robotics from past to present-a historical perspective," in Advances in Rehabilitation Robotics, pp. 25-44, Springer, New York, NY, USA, 2004.

[98] A. Waldner, C. Werner, and S. Hesse, "Robot assisted therapy in neurorehabilitation," Europa Medicophysica, vol. 44, supplement 1, pp. 1-3, 2008.

[99] J. L. Dallaway, R. D. Jackson, and P. H. A. Timmers, "Rehabilitation robotics in Europe," IEEE Transactions on Rehabilitation Engineering, vol. 3, no. 1, pp. 35-45, 1995.

[100] M. Busnel, R. Cammoun, F. Coulon-Lauture, J. M. Détriché, G. Le Claire, and B. Lesigne, "The robotized workstation "MASTER" for users with tetraplegia: description and evaluation," Journal of Rehabilitation Research and Development, vol. 36, no. 3, pp. 217-229, 1999.

[101] M. Ceccarelli, "Problems and issues for service robots in new applications," International Journal of Social Robotics, vol. 3, no. 3, pp. 299-312, 2011.

[102] M. J. H. Lum, D. C. W. Friedman, G. Sankaranarayanan et al., "The RAVEN: design and validation of a telesurgery system," International Journal of Robotics Research, vol. 28, no. 9, pp. 1183-1197, 2009.

[103] A. Simorov, R. Otte, C. Kopietz, and D. Oleynikov, "Review of surgical robotics user interface: what is the best way to 
control robotic surgery?" Surgical Endoscopy, vol. 26, no. 8, pp. 2117-2125, 2012.

[104] C. Kuo, J. Dai, and P. Dasgupta, "Kinematic design considerations for minimally invasive surgical robots: an overview," The International Journal of Medical Robotics and Computer Assisted Surgery, vol. 8, no. 2, pp. 127-145, 2012.

[105] C. Wagner, N. Stylopoulos, and R. Howe, "The role of force feedback in surgery: analysis of blunt dissection," in Proceedings of the 10th Symposium on Haptic Interfaces for Virtual Environment and Teleoperator Systems, vol. 2002, Citeseer, 2002.

[106] M. Tavakoli, R. V. Patel, and M. Moallem, "Haptic interaction in robot-assisted endoscopic surgery: a sensorized endeffector," The International Journal of Medical Robotics and Computer Assisted Surgery, vol. 1, no. 2, pp. 53-63, 2005.

[107] A. M. Okamura, "Haptic feedback in robot-assisted minimally invasive surgery," Current Opinion in Urology, vol. 19, no. 1, pp. 102-107, 2009.

[108] U. Hagn, R. Konietschke, A. Tobergte et al., "DLR MiroSurge: a versatile system for research in endoscopic telesurgery," International Journal of Computer Assisted Radiology and Surgery, vol. 5, no. 2, pp. 183-193, 2010.

[109] S. Thielmann, U. Seibold, R. Haslinger et al., "MICA-a new generation of versatile instruments in robotic surgery," in Proceedings of the 23rd IEEE/RSJ International Conference on Intelligent Robots and Systems (IROS '10), pp. 871-878, October 2010.

[110] R. Konietschke, T. Ortmaier, H. Weiss, G. Hirzinger, and R. Engelke, "Manipulability and accuracy measures for a medical robot in minimally invasive surgery," in Advances in Robot Kinematics, 2004.

[111] G. Sutherland, P. McBeth, and D. Louw, "Neuroarm: an mr compatible robot for microsurgery," in International Congress Series, vol. 1256, pp. 504-508, Elsevier, Amsterdam, The Netherland, 2003.

[112] M. J. Lang, A. D. Greer, and G. R. Sutherland, "Intraoperative robotics: NeuroArm," Intraoperative Imaging, vol. 109, pp. 231-236, 2011.

[113] D. Stoianovici, D. Song, D. Petrisor et al., “'MRI Stealth' robot for prostate interventions," Minimally Invasive Therapy and Allied Technologies, vol. 16, no. 4, pp. 241-248, 2007.

[114] P. Garcia, J. Rosen, C. Kapoor et al., "Trauma pod: a semiautomated telerobotic surgical system," International Journal of Medical Robotics and Computer Assisted Surgery, vol. 5, no. 2, pp. 136-146, 2009.

[115] S. G. Yuen, P. M. Novotny, and R. D. Howe, "Quasiperiodic predictive filtering for robot-assisted beating heart surgery," in Proceedings of the IEEE International Conference on Robotics and Automation (ICRA '08), pp. 3875-3880, May 2008.

[116] N. Patronik, C. Riviere, S. El Qarra, and M. Zenati, "The heartlander: a novel epicardial crawling robot for myocardial injections," in International Congress Series, vol. 1281, pp. 735-739, Elsevier, Amsterdam, The Netherland, 2005.

[117] D. Moral Del Agua, N. A. Wood, and C. N. Riviere, "Improved synchronization of heartlander locomotion with physiological cycles," in Proceedings of the 37th Annual Northeast Bioengineering Conference (NEBEC '11), April 2011.

[118] T. Wortman, A. Meyer, O. Dolghi et al., "Miniature surgical robot for laparoendoscopic single-incision colectomy," Surgical Endoscopy, vol. 26, pp. 727-731, 2012.
[119] Y. Hayashi, H. Yamamoto, T. Yano, and K. Sugano, "Review: diagnosis and management of mid-gastrointestinal bleeding by double-balloon endoscopy," Therapeutic Advances in Gastroenterology, vol. 2, no. 2, pp. 109-117, 2009.

[120] A. Van Gossum, M. M. Navas, I. Fernandez-Urien et al., "Capsule endoscopy versus colonoscopy for the detection of polyps and cancer," The New England Journal of Medicine, vol. 361, no. 3, pp. 264-270, 2009.

[121] D. Cassilly, S. Kantor, L. C. Knight et al., "Gastric emptying of a non-digestible solid: assessment with simultaneous SmartPill $\mathrm{pH}$ and pressure capsule, antroduodenal manometry, gastric emptying scintigraphy," Neurogastroenterology and Motility, vol. 20, no. 4, pp. 311-319, 2008.

[122] C. Mc Caffrey, O. Chevalerias, C. O'Mathuna, and K. Twomey, "Swallowable-capsule technology," IEEE Pervasive Computing, vol. 7, no. 1, pp. 23-29, 2008.

[123] A. Moglia, A. Menciassi, and P. Dario, "Recent patents on wireless capsule endoscopy," Recent Patents on Biomedical Engineering, vol. 1, no. 1, pp. 24-33, 2008.

[124] M. C. Roco, "Nanotechnology: convergence with modern biology and medicine," Current Opinion in Biotechnology, vol. 14, no. 3, pp. 337-346, 2003.

[125] M. Copot, A. Popescu, I. Lung, and A. Moldovanu, "Achievements and perspectives in the field of nanorobotics," The Romanian Review Precision Mechanics, Optics and Mechatronics, vol. 19, no. 36, pp. 61-66, 2009.

[126] R. A. Freitas, "What is nanomedicine?" Nanomedicine: Nanotechnology, Biology, and Medicine, vol. 1, no. 1, pp. 2-9, 2005.

[127] L. Zhang, J. J. Abbott, L. Dong, B. E. Kratochvil, D. Bell, and B. J. Nelson, "Artificial bacterial flagella: fabrication and magnetic control," Applied Physics Letters, vol. 94, no. 6, Article ID 064107, 2009.

[128] G. Kósa, M. Shoham, and M. Zaaroor, "Propulsion of a swimming micro medical robot," in Proceedings of the IEEE International Conference on Robotics and Automation (ICRA '05), pp. 1327-1331, April 2005.

[129] G. Dogangil, O. Ergeneman, J. J. Abbott et al., "Toward targeted retinal drug delivery with wireless magnetic microrobots," in Proceedings of the IEEE/RSJ International Conference on Intelligent Robots and Systems (IROS '08), pp. 19211926, September 2008.

[130] H. Li, J. Tan, and M. Zhang, "Dynamics modeling and analysis of a swimming microrobot for controlled drug delivery," IEEE Transactions on Automation Science and Engineering, vol. 6, no. 2, pp. 220-227, 2009.

[131] T. Ebefors, J. Mattsson, E. Kalvesten, and G. Stemme, "A walking silicon micro-robot," in Proceedings of the 10th International Conference on Solid-State Sensors and Actuators (Transducers '99), pp. 1202-1205, 1999.

[132] P. Dumpuri, L. W. Clements, B. M. Dawant, and M. I. Miga, "Model-updated image-guided liver surgery: preliminary results using surface characterization," Progress in Biophysics and Molecular Biology, vol. 103, no. 2-3, pp. 197-207, 2010.

[133] G. Brandt, A. Zimolong, L. Carrât et al., "CRIGOS: a compact robot for image-guided orthopedic surgery," IEEE Transactions on Information Technology in Biomedicine, vol. 3, no. 4, pp. 252-260, 1999. 

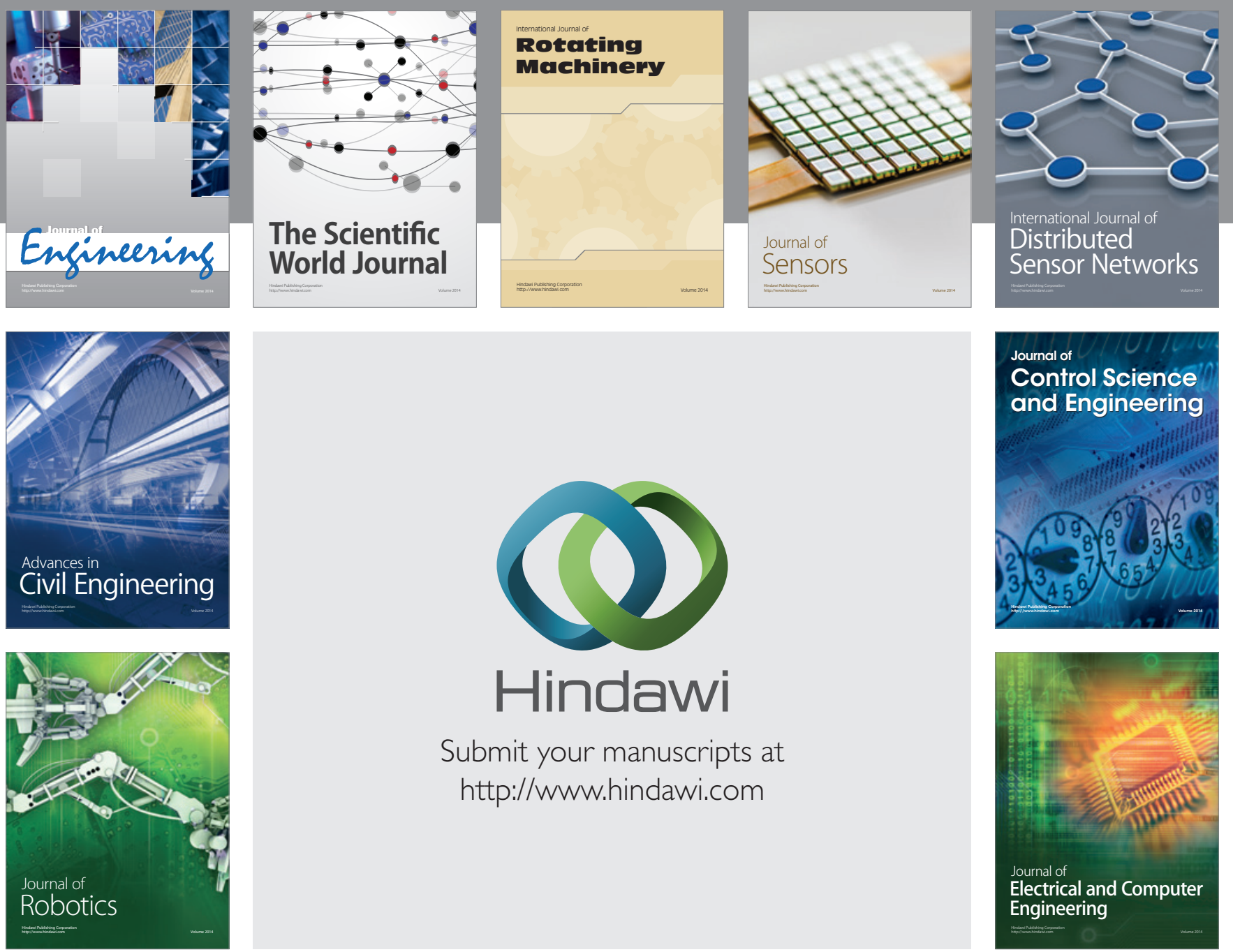

Submit your manuscripts at

http://www.hindawi.com
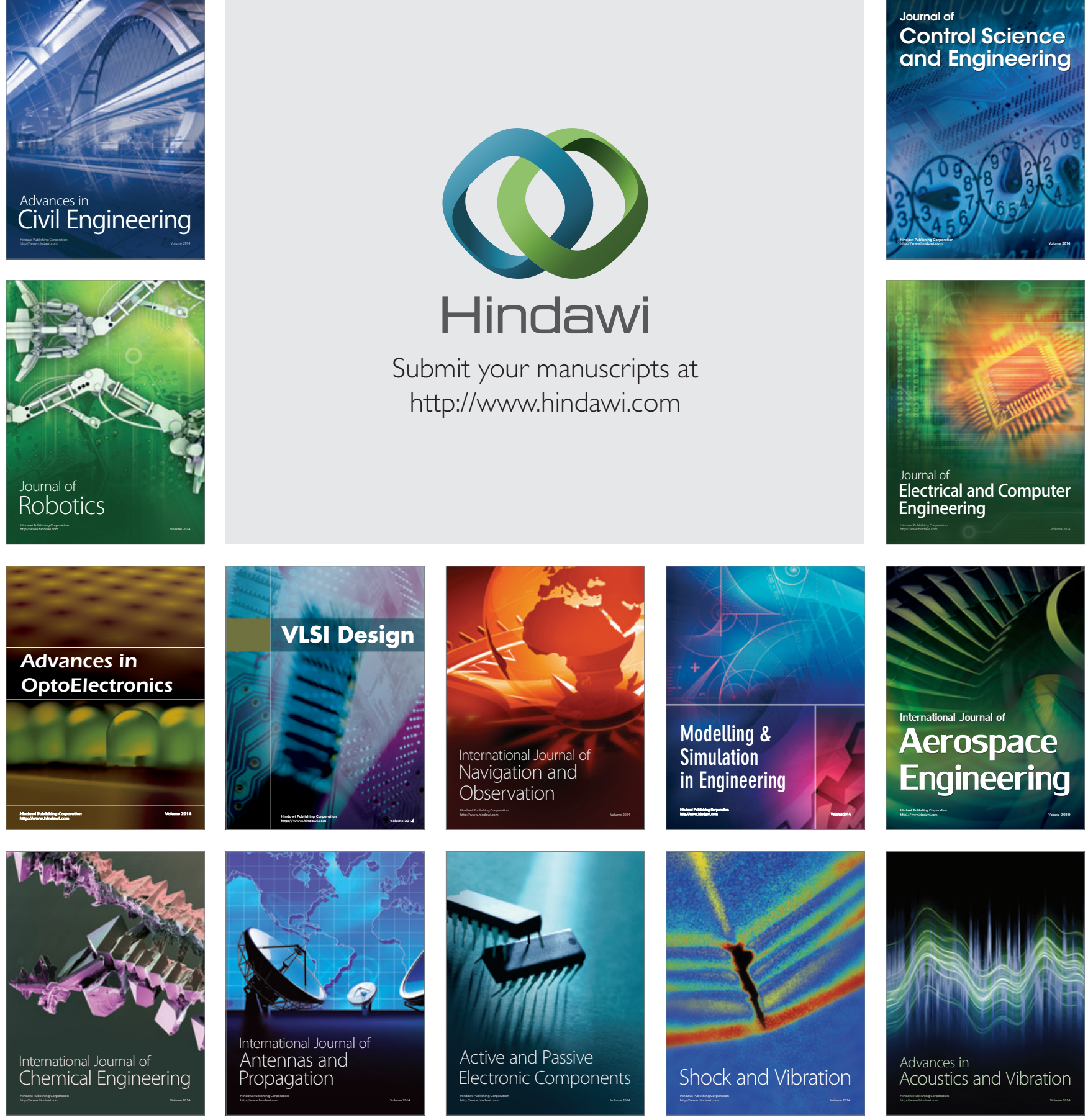